\title{
Propagation Characteristics of a Twisted Cosine-Gaussian Correlated Radially Polarized Beam
}

\author{
Jipeng Zhang ${ }^{1}$, Jing Wang ${ }^{1}$, Hongkun Huang ${ }^{1}$, Haiyan Wang ${ }^{1}$, Shijun Zhu ${ }^{1,2, *(\mathbb{D}}$, Zhenhua Li ${ }^{1}$ \\ and Jian $\mathrm{Lu}^{1}$ \\ 1 Department of Information Physics and Engineering, Nanjing University of Science and Technology, \\ Nanjing 210094, China; m18362960516_1@163.com (J.Z.); wangjingnjust@163.com (J.W.); \\ 13770314598@163.com (H.H.); njustwhy@njust.edu.cn (H.W.); lizhenhua@njust.edu.cn (Z.L.); \\ lujian@njust.edu.cn (J.L.) \\ 2 School of Optoelectronic Science and Engineering, Soochow University, Suzhou 215006, China \\ * Correspondence: shijunzhu@njust.edu.cn
}

Received: 31 July 2018; Accepted: 26 August 2018; Published: 29 August 2018

Featured Application: The results provide a useful guideline for manipulation of novel vector structure beams by using twist phases and customized correlation functions, and promote important potential applications, ranging from beam shaping, optical tweezers, optical imaging, and free space optical communications.

\begin{abstract}
Recently, partially coherent beams with twist phases have attracted growing interest due to their nontrivial dynamic characteristics. In this work, the propagation characteristics of a twisted cosine-Gaussian correlated radially polarized beam such as the spectral intensity, the spectral degree of coherence, the degree of polarization, the state of polarization, and the spectral change are investigated in detail. Due to the presence of the twisted phase, the beam spot, the degree of coherence, and the state of polarization experience rotation during transmission, but the degree of polarization is not twisted. Meanwhile, although their rotation speeds closely depend on the value of the twist factor, they all undergo a rotation of $\pi / 2$ when they reach the focal plane. Furthermore, the effect of the twist phase on the spectral change is similar to the coherence, which is achieved by modulating the spectral density distribution during transmission. The twist phase opens up a useful guideline for manipulation of novel vector structure beams and enriches potential applications in the field of beam shaping, optical tweezers, optical imaging, and free space optical communications.
\end{abstract}

Keywords: partially coherent; radially polarized; twist phase; state of polarization

\section{Introduction}

It is well-known that the coherence properties play an important role in determining the spatial behaviors of light beams under propagation [1-10]. For instance, there is a Fourier reciprocal relationship between the initial coherence and the far field intensity distribution [1,2]. Also, it has been proven that the source coherence can apparently influence the evolution behaviors of the degree of polarization and state of polarization of light beams after propagation, even in free space $[7,8]$. These results contradict the common belief that polarization is propagation invariant. However, most of the studies concerning the spatial coherence sources are restricted to the so-called Schell-model (SM) correlations, where the spectral degree of coherences obey Gaussian distributions [1-3]. In recent years, based on the nonnegative definiteness criteria of the cross-spectral density (CSD), Gori and Santarsiero proposed a sufficient condition for devising genuine correlation functions and quickly extended to the vector cases [11,12]. Manipulation of nontrivial correlation structures to produce 
prescribed structure beams with tailored intensity, polarization, and phase, and to explore novel spatial behaviors has seen a rapid growth of interest due to their important applications in optical imaging, optical tweezers, free-space optical communications, laser radar, and remote sensing [13-33].

In previous decades, much prominence has been given to radially polarized beams because of their nontrivial properties and potential applications such as lithography, display technologies, optical trapping, optical data storage, and confocal microscopy [34-42]. A partially coherent radially polarized beam was introduced as a natural extension of a coherent cylindrical vector beam. Paraxial and non-paraxial properties of partially coherent radially polarized beams have been investigated in detail [43-49]. Statistical properties of partially coherent radially polarized beams in random media such as a turbulent atmosphere and ocean turbulence have been studied [50,51]. It is demonstrated that partially coherent radially polarized beams are more effective at resisting signal distortion caused by turbulence than general linearly polarized partially coherent beams. Moreover, by manipulating the correlation structures of source beams, the ability to generate tunable partially coherent radially polarized array beams was demonstrated [22-26].

In 1993, Simon and Mukunda proposed that a partially coherent beam has the ability to carry a new type of phase-twist phase, and this was later generated experimentally by Friberg et al. [52,53]. Recently, on the basis of the nonnegative constraint of the CSD, Gori et al. introduced a new method to design twisted sources endowed with circular or rectangular symmetry $[54,55]$. Due to the existence of the twist phase, twisted partially coherent beams not only have the ability to carry orbital angular momentum, but also have better anti-turbulence self-repairing capabilities, which can find applications in optical tweezers, optical imaging, and optical communications [56-64]. Besides, owing to their nontrivial angular momentum mechanical properties, twisted partially coherent beams are receiving increasing attention $[65,66]$. In this paper, our aim is to study the focusing properties of a new class of partially coherent radially polarized beams with a nontrivial cosine-Gaussian correlation function and a twisted phase. The effects of the twist phase on the spectral intensity, the spectral degree of coherence, the degree of polarization, the state of polarization, and the spectral change are investigated in detail. The results yield a useful guideline for manipulation of novel vector structure beams by using twist phases and customized correlation functions, and promote important potential applications, ranging from beam shaping, optical tweezers, optical imaging, and free space optical communications.

\section{Theory}

It is known that the vector electric field of a radially polarized beam can be described as the coherent superposition of $\mathrm{TEM}_{01}$ Laguerre-Gaussian modes oriented along the $x$ axis and a $\mathrm{TEM}_{10}$ oriented along the $y$ axis at $z=0$ [43-48].

$$
\mathbf{E}(\mathbf{r})=E_{x}(\mathbf{r}) \mathbf{e}_{x}+E_{y}(\mathbf{r}) \mathbf{e}_{y}=\frac{x}{w_{0}} \exp \left(-\frac{\mathbf{r}^{2}}{w_{0}^{2}}\right) \mathbf{e}_{x}+\frac{y}{w_{0}} \exp \left(-\frac{\mathbf{r}^{2}}{w_{0}^{2}}\right) \mathbf{e}_{y},
$$

where $\mathbf{r}=\left(x^{2}+y^{2}\right)^{1 / 2}$ denotes the transversal distance from the beam center and $w_{0}$ is the transverse beam size. For a vector partially coherent beam in space-frequency domain, the second-order correlation properties of a fluctuating light beam can be completely described by the CSD matrix $\overline{\mathbf{W}}\left(\mathbf{r}_{1}, \mathbf{r}_{2}\right)$ with elements $W\left(\mathbf{r}_{1}, \mathbf{r}_{2}\right)=\left\langle E_{\alpha}^{*}\left(\mathbf{r}_{1}\right) E_{\beta}\left(\mathbf{r}_{2}\right\rangle, \alpha, \beta \in\{x, y\} . \mathbf{r}_{1}\right.$ and $\mathbf{r}_{2}$ denote two arbitrary points with position vectors in the source plane. For brevity, we omit the explicit dependence of the considered quantities on frequency $\omega$. The asterisk denotes the complex conjugate and the angular brackets represent average over the statistical ensemble. For a partially coherent radially polarized beam, the elements of the CSD matrix are described as [46]:

$$
W_{\alpha \beta}\left(\mathbf{r}_{1}, \mathbf{r}_{2}\right)=\frac{\alpha_{1} \beta_{2}}{w_{0}^{2}} \exp \left(-\frac{\mathbf{r}_{1}^{2}+\mathbf{r}_{2}^{2}}{w_{0}^{2}}\right) g_{\alpha \beta}\left(\mathbf{r}_{1}, \mathbf{r}_{2}\right), \quad(\alpha, \beta \in\{x, y\}),
$$


where $g$ denotes the degree of coherence.

It is important to note that the coherence structure is independent of spectral density profile. So, one can independently modulate the coherence function without affecting the spectral density of random sources [54]. Here, let us consider the coherence function of the initial beam to have a twisted cosine-Gaussian correlated (CGC) function [25,54]:

$$
g_{\alpha \beta}\left(\mathbf{r}_{1}, \mathbf{r}_{2}\right)=\exp \left[-\frac{\left(\mathbf{r}_{1}-\mathbf{r}_{2}\right)^{2}}{2 \delta_{0}^{2}}\right] \cos \left[\frac{n \sqrt{\pi}\left(x_{1}-x_{2}\right)}{\delta_{0}}\right] \cos \left[\frac{n \sqrt{\pi}\left(y_{1}-y_{2}\right)}{\delta_{0}}\right] \exp \left[-i k \mu\left(x_{1} y_{2}-x_{2} y_{1}\right)\right],
$$

where $\delta_{0}$ denotes coherence parameter, $n$ is a positive beam order parameter, and $\mu$ represents the twist phase. When $n=0$, a twisted CGC radially polarized beam reduces to a conventional Schell-model (SM) radially polarized beam [44-48]. Using Mercer's expansion, it is proven that the realizability condition for generation of such twisted random source coincides with SM sources. As a new class of twisted non-uniformly correlated vector beams, a possible experimental approach for generating twisted CGC radially polarized beams can be achieved by using a spatial light modulator and an astigmatic optical lens system $[20,25,53]$. In [25], we have demonstrated an experiment for generating a CGC correlated radially polarized beam. One may further produce a twisted CGC radially polarized beam by passing a CGC correlated radially polarized beam through an astigmatic optical lens system [53].

Within the framework of paraxial approximation, the elements of the CSD matrix of a twisted CGC radially polarized beam through an ABCD optical system can be written as [2]:

$$
\begin{aligned}
W_{\alpha \beta}\left(\mathbf{r}_{1}^{\prime}, \mathbf{r}_{2}^{\prime}\right) & =\frac{k^{2}}{4 \pi^{2} B^{2}} \int_{-\infty}^{\infty} \int_{-\infty}^{\infty} \int_{-\infty}^{\infty} \int_{-\infty}^{\infty} W_{\alpha \beta}\left(\mathbf{r}_{1}, \mathbf{r}_{2}\right) \exp \left[-\frac{i k}{2 B}\left(A \mathbf{r}_{1}{ }^{2}-2 \mathbf{r}_{1} \cdot \mathbf{r}_{1}^{\prime}+D \mathbf{r}_{1}^{\prime 2}\right)\right] \\
& \times \exp \left[\frac{i k}{2 B}\left(A \mathbf{r}_{2}^{2}-2 \mathbf{r}_{2} \cdot \mathbf{r}_{2}^{\prime}+D \mathbf{r}_{2}^{\prime 2}\right)\right] d^{2} \mathbf{r}_{1} d^{2} \mathbf{r}_{2} .
\end{aligned}
$$

On substituting Equations (2) and (3) into Equation (4), after some algebra the elements of the CSD matrix of a twisted CGC radially polarized beam in the output plane turn out to be:

$$
\begin{aligned}
& W_{x x}\left(\mathbf{r}_{1}^{\prime}, \mathbf{r}_{2}^{\prime}\right)=\frac{k^{2}}{64 w_{0}^{2} B^{2} N_{1}^{2}\left(N_{2}-\Omega\right)^{2}} \exp \left[-\frac{i k D}{2 B}\left(\mathbf{r}_{1}^{\prime 2}-\mathbf{r}_{2}^{\prime 2}\right)\right] \\
& \times \sum_{a_{1}= \pm a} \sum_{a_{2}= \pm a}\left[\xi_{u 11}\left(\xi_{u 21}+i \Pi_{1}\right)+\delta_{0}^{-2}-\frac{\left(i \xi_{u 21}-\Pi_{1}\right)^{2}}{2 \delta_{0}^{2}\left(N_{2}-\Omega\right)}+\frac{k \mu\left(\xi_{u 21}+i \Pi_{1}\right)\left(i \xi_{v 22}-\Pi_{2}\right)}{2\left(N_{2}-\Omega\right)}\right] \\
& \times \exp \left[-\frac{\xi_{v 12}^{2}+\xi_{u 11}^{2}}{4 N_{1}}+\frac{\left(i \tilde{\zeta}_{u 21}-\Pi_{1}\right)^{2}+\left(i \xi_{v 22}-\Pi_{2}\right)^{2}}{4\left(N_{2}-\Omega\right)}\right], \\
& W_{y y}\left(\mathbf{r}_{1}^{\prime}, \mathbf{r}_{2}^{\prime}\right)=\frac{k^{2}}{64 w_{0}^{2} B^{2} N_{1}^{2}\left(N_{2}-\Omega\right)^{2}} \exp \left[-\frac{i k D}{2 B}\left(\mathbf{r}_{1}^{\prime 2}-\mathbf{r}_{2}^{\prime 2}\right)\right] \\
& \times \sum_{a_{1}= \pm a} \sum_{a_{2}= \pm a}\left[\xi_{v 12}\left(\xi_{v 22}+i \Pi_{2}\right)+\delta_{0}^{-2}-\frac{\left(i \xi_{v 22}-\Pi_{2}\right)^{2}}{2 \delta_{0}^{2}\left(N_{2}-\Omega\right)}-\frac{k \mu\left(\xi_{v 22}+i \Pi_{2}\right)\left(i \xi_{u 21}-\Pi_{1}\right)}{2\left(N_{2}-\Omega\right)}\right] \\
& \times \exp \left[-\frac{\xi_{v 12}^{2}+\xi_{u 11}^{2}}{4 N_{1}}+\frac{\left(i \xi_{v 22}-\Pi_{2}\right)^{2}+\left(i \tilde{\zeta}_{u 21}-\Pi_{1}\right)^{2}}{4\left(N_{2}-\Omega\right)}\right], \\
& W_{x y}\left(\mathbf{r}_{1}^{\prime}, \mathbf{r}_{2}^{\prime}\right)=\frac{k^{2}}{64 w_{0}^{2} B^{2} N_{1}^{2}\left(N_{2}-\Omega\right)^{2}} \exp \left[-\frac{i k D}{2 B}\left({\mathbf{r}_{1}^{\prime}}^{2}-\mathbf{r}_{2}^{\prime 2}\right)\right] \\
& \times \sum_{a_{1}= \pm a} \sum_{a_{2}= \pm a}\left[\xi_{u 11}\left(\xi_{v 22}+i \Pi_{2}\right)+i k \mu+i k \mu \frac{\left(i \xi_{v 22}-\Pi_{2}\right)^{2}}{2\left(N_{2}-\Omega\right)}-\frac{\left(i \xi_{v 22}-\Pi_{2}\right)\left(i \xi_{u 21}-\Pi_{1}\right)}{2 \delta_{0}^{2}\left(N_{2}-\Omega\right)}\right] \\
& \times \exp \left[-\frac{\xi^{2} v 12+\tau^{2} u 11}{4 N_{1}}+\frac{\left(i \xi_{v 22}-\Pi_{2}\right)^{2}+\left(i \xi_{u 21}-\Pi_{1}\right)^{2}}{4\left(N_{2}-\Omega\right)}\right] \text {. }
\end{aligned}
$$

where

$$
\begin{aligned}
& a=\sqrt{2 \pi} n / \delta_{0}, N_{1}=\frac{1}{4 w_{0}^{2}}+\frac{1}{2 \delta_{0}^{2}}+\frac{i k A}{2 B}, N_{2}=\frac{1}{4 w_{0}^{2}}+\frac{1}{2 \delta_{0}^{2}}-\frac{i k A}{2 B}, \\
& \xi_{u 11}=k x_{1}^{\prime} / B+a_{1}, \xi_{u 21}=k x_{2}^{\prime} / B+a_{1}, \xi_{v 12}=k y_{1}^{\prime} / B+a_{2}, \xi_{v 22}=k y_{2}^{\prime} / B+a_{2}, \\
& \Omega=\frac{1}{4 N_{1} \delta_{0}^{4}}-\frac{k^{2} \mu^{2}}{4 N_{1}}, \Pi_{1}=\frac{i \xi_{u 11}}{2 N_{1} \delta_{0}^{2}}-\frac{\xi_{v 12} k \mu}{2 N_{1}}, \Pi_{2}=\frac{i \xi_{v 12}}{2 N_{1} \delta_{0}^{2}}+\frac{\xi_{u 11} k \mu}{2 N_{1}} .
\end{aligned}
$$


On the basis of the CSD matrix of an electromagnetic beam, there are three important fundamental statistical characteristics that can be defined as:

the spectral density

$$
S\left(\mathbf{r}^{\prime}\right)=W_{x x}\left(\mathbf{r}^{\prime}, \mathbf{r}^{\prime}\right)+W_{y y}\left(\mathbf{r}^{\prime}, \mathbf{r}^{\prime}\right)
$$

the spectral degree of coherence (DOC)

$$
\eta\left(\mathbf{r}_{1}^{\prime}, \mathbf{r}_{2}^{\prime}\right)=\frac{\operatorname{Tr}\left[\overline{\mathbf{W}}\left(\mathbf{r}_{1}^{\prime}, \mathbf{r}_{2}^{\prime}\right)\right]}{\left(\operatorname{Tr}\left[\overline{\mathbf{W}}\left(\mathbf{r}_{1}^{\prime}, \mathbf{r}_{1}^{\prime}\right)\right] \operatorname{Tr}\left[\overline{\mathbf{W}}\left(\mathbf{r}_{2}^{\prime}, \mathbf{r}_{2}^{\prime}\right)\right]\right)^{1 / 2}}
$$

and the degree of polarization (DOP)

$$
P\left(\mathbf{r}^{\prime}\right)=\left(1-\frac{4 \operatorname{Det}\left[\overline{\mathbf{W}}\left(\mathbf{r}^{\prime}, \mathbf{r}^{\prime}\right)\right]}{\left(\operatorname{Tr}\left[\overline{\mathbf{W}}\left(\mathbf{r}^{\prime}, \mathbf{r}^{\prime}\right)\right]\right)^{2}}\right)^{1 / 2}
$$

In Equations (9)-(11), Tr represents the trace and Det denotes the determinant. It should be noted that there is another definition of the spectral DOC for electromagnetic beams introduced by Tervo et al., which is also widely used [9].

\section{Numerical Results}

Now, we numerically analyze the focusing properties of a twisted CGC radially polarized beam with the help of the theoretical results derived above. Let us consider the source beam passes through a thin lens with focal length $f$ and then arrives at the receiver plane. The ray matrix of such optical system reads as:

$$
\left(\begin{array}{ll}
A & B \\
C & D
\end{array}\right)=\left(\begin{array}{ll}
1 & z \\
0 & 1
\end{array}\right)\left(\begin{array}{cc}
1 & 0 \\
-1 / f & 1
\end{array}\right)\left(\begin{array}{ll}
1 & f \\
0 & 1
\end{array}\right)=\left(\begin{array}{cc}
1-z / f & f \\
-1 / f & 0
\end{array}\right) .
$$

The global parameters used in the following calculations are set as $\lambda=632.8 \mathrm{~nm}, w_{0}=1 \mathrm{~mm}$, $\delta_{0}=0.2 \mathrm{~mm}, \mu=0.001 \mathrm{~mm}^{-1}$, and $f=150 \mathrm{~mm}$, unless different values are specified. In Figure 1 , we depict the normalized spectral density distribution and the corresponding cross line of a twisted CGC radially polarized beam focused by a thin lens at several propagation distances for different values of $n$. For the case of $n \neq 0$, it is shown that the spectral density gradually decomposes into a four-beamlets array distribution, and the distance between each beamlet grows as the beam order $n$ increases. Moreover, one clearly sees that the spectral density distribution of a twisted CGC radially polarized beam in the focal plane is significantly different from that in the absence of the twist phase. As expected, for a CGC radially polarized beam without the twist phase, there is a Fourier-like relation between the spectral density in the focal plane and the initial correlation function [25]. However, for a twisted CGC radially polarized beam, this genuine reciprocal relationship disappears due to the existence of the twist phase, see Figure 1a3,b3,c3. The reason for this is that the twist phase leads to a beam astigmatism during diffraction, which causes a focal shift. 

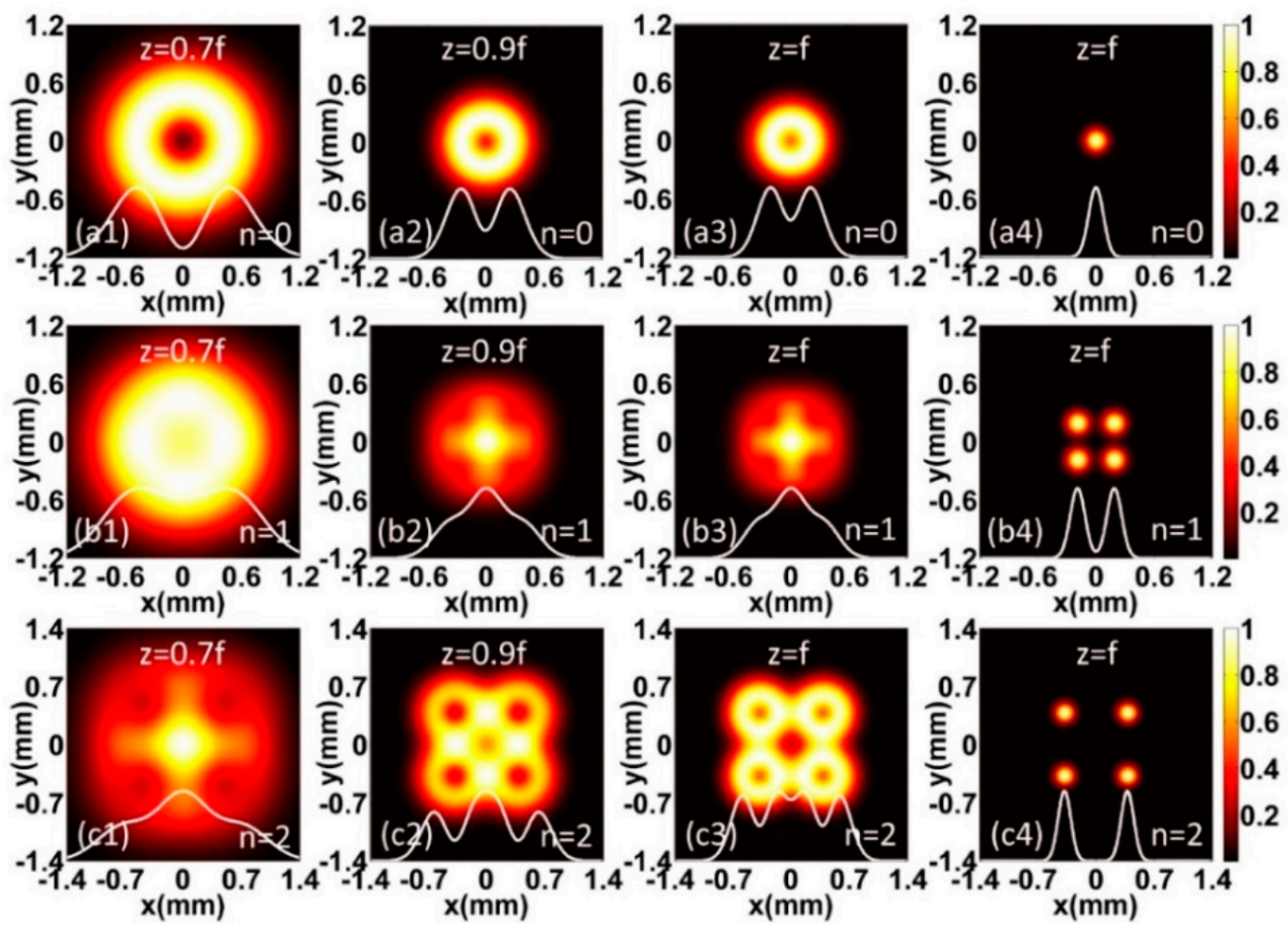

Figure 1. Normalized spectral density distribution and the corresponding cross line of a focused twisted cosine-Gaussian correlated (CGC) radially polarized beam at different propagating distances for different values of $n$. For (a1-a3), (b1-b3), (c1-c3) $\mu=0.001 \mathrm{~mm}^{-1} ;(\mathbf{a} 4, \mathbf{b} 4, \mathbf{c} 4) \mu=0 \mathrm{~mm}^{-1}$.

In order to study the spectral density distribution of polarization components $W_{x x}\left(\mathbf{r}^{\prime}, \mathbf{r}^{\prime}\right)$ and $W_{y y}\left(\mathbf{r}^{\prime}, \mathbf{r}^{\prime}\right)$, Figure 2 plots the normalized polarized spectral density components and the corresponding cross lines at several propagation distances with $n=2$. It is clearly seen that the spectral density not only splits during spreading, but also each spectral density component undergoes a rotation of $\pi / 2$ within the focal length. In addition, for a CGC radially polarized beam, see Figure $2 b 4, c 4$, one finds that the two orthogonal spectral density components have the same distribution in the focal plane.

Figure 3 shows the modulus of the spectral DOC and the corresponding cross line at different propagating distances for different values of $n$. One finds that the array structure of the spectral DOC becomes more complex with the growth of the order parameter $n$. Similar to the spectral density, it is clearly seen that the spectral DOC rotates during the spreading and also experience a rotation of $\pi / 2$ when they reach the focal plane. At the same time, it is found that the rotation speed depends only on the twist phase, see Figure 3b1-b3,c1-c3. In addition, due to the existence of the twist phase, the Fourier reciprocal relationship between the spectral DOC in the focal plane and the initial spectral intensity no longer exists. Thus, the spectral DOC of a twisted CGC radially polarized beam in the focal plane is quite different from that without the twist phase [25]. 

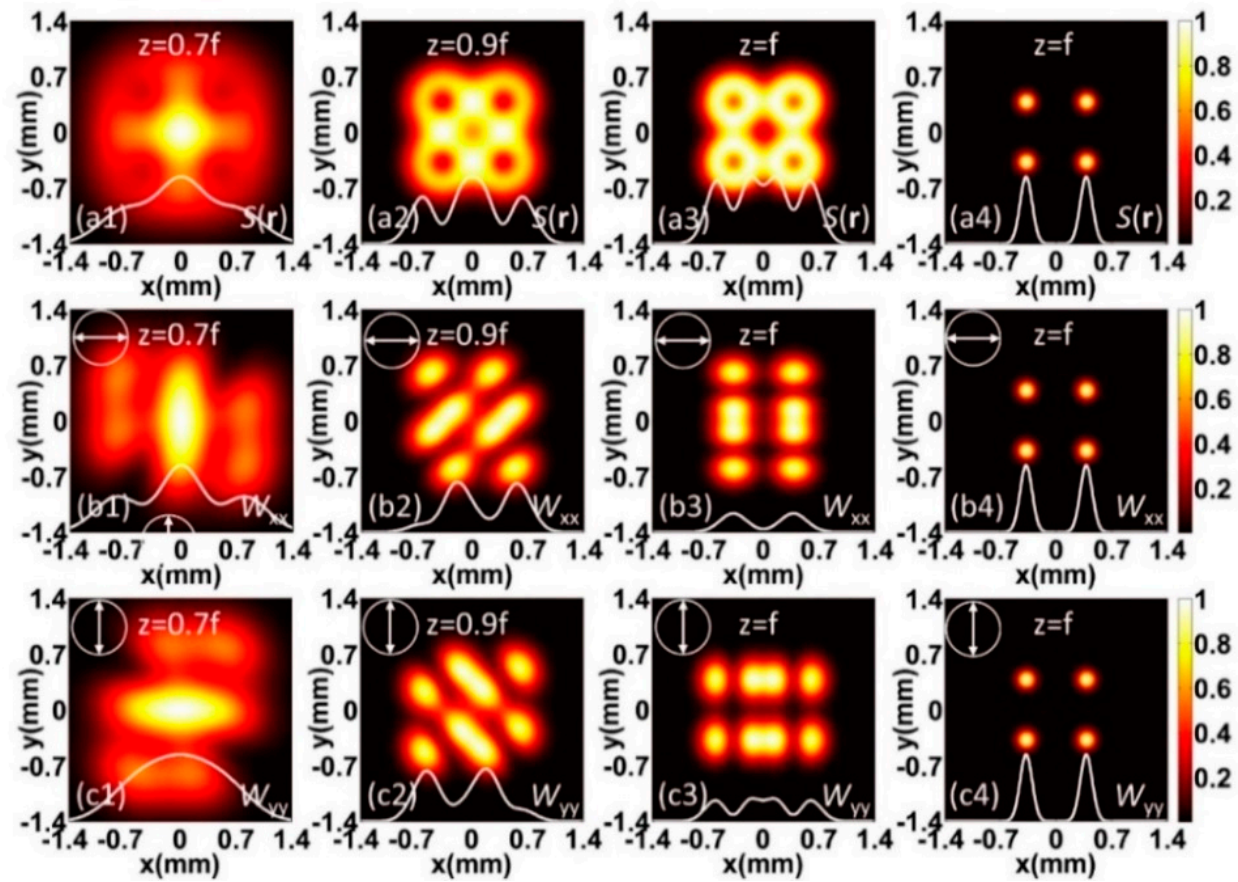

Figure 2. Normalized spectral intensity distribution, polarized spectral intensity components $W_{x x}\left(\mathbf{r}^{\prime}, \mathbf{r}^{\prime}\right)$ and $W_{y y}\left(\mathbf{r}^{\prime}, \mathbf{r}^{\prime}\right)$, and the corresponding cross line of a focused twisted CGC radially polarized beam at different propagating distances with $n=2$. For (a1-a3), (b1-b3), (c1-c3) $\mu=0.001 \mathrm{~mm}^{-1} ;(\mathbf{a} 4, \mathbf{b} 4, \mathbf{c 4}) \mu=0 \mathrm{~mm}^{-1}$.
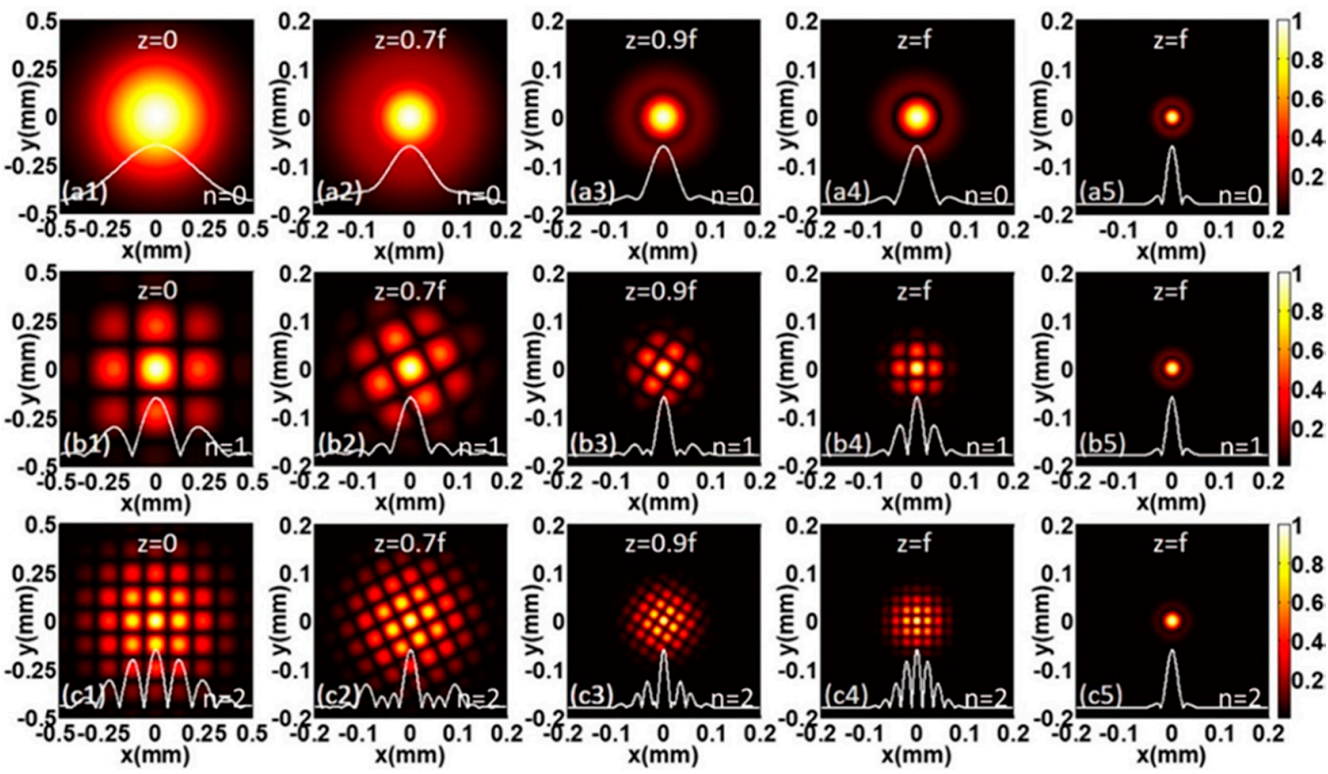

Figure 3. Modulus of the spectral degree of coherence (DOC) and the corresponding cross line of a focused twisted CGC radially polarized beam at different propagation distances for different values of $n$. For (a1-a4), (b1-b4), (c1-c4) $\mu=0.001 \mathrm{~mm}^{-1} ;(\mathbf{a} 5, \mathbf{b} 5, \mathbf{c} 5) \mu=0 \mathrm{~mm}^{-1}$.

Figure 4 illustrates the effect of the twist phase on the spectral DOC. As is seen from Figure 4, the rotation speed is nonlinear and closely depends on the value of the twist factor. However, when they reach the focal plane, they all undergo a rotation of $\pi / 2$, regardless of the value of the twist phase. Furthermore, it is of interest to see that the spectral DOC spot becomes larger as the twist factor increases due to the beam divergence caused by the twist phase. 

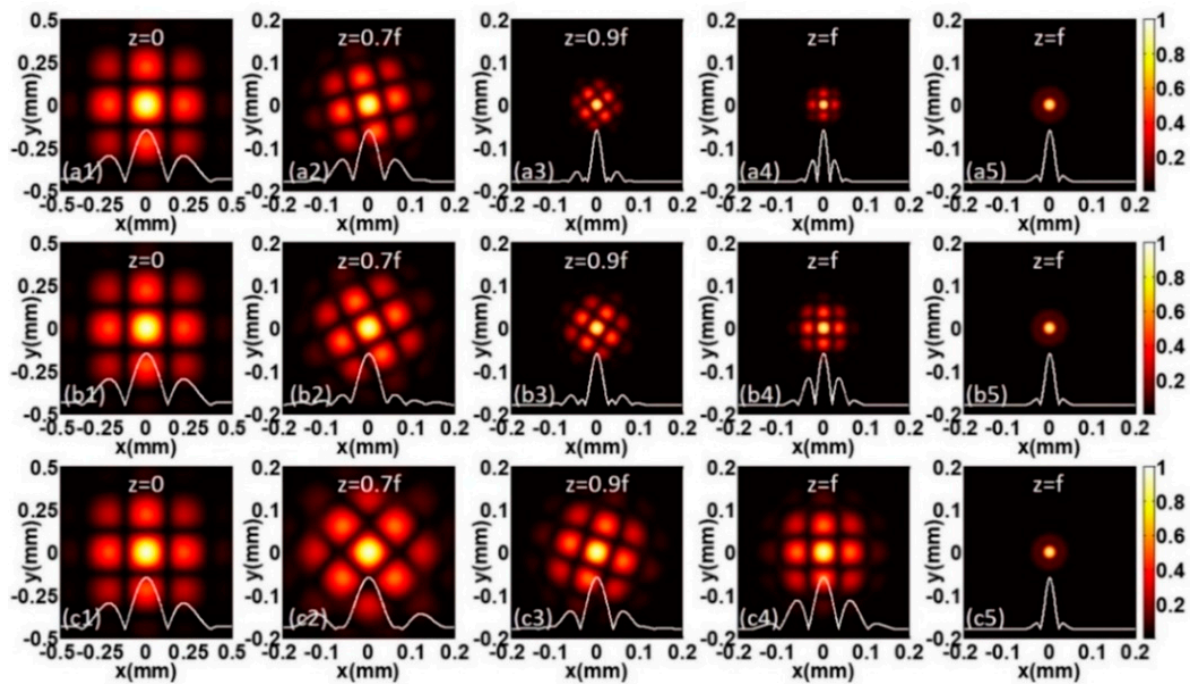

Figure 4. Modulus of the spectral DOC and the corresponding cross line of focused twisted CGC radially polarized beam at different propagation distances for different values of the twist phase. For (a1-a4) $\mu=0.0005 \mathrm{~mm}^{-1}$; (b1-b4) $\mu=0.001 \mathrm{~mm}^{-1}$; (c1-c4) $\mu=0.002 \mathrm{~mm}^{-1} ;(\mathbf{a} 5, \mathbf{b} 5, \mathbf{c 5}) \mu=0 \mathrm{~mm}^{-1}$.

In order to learn more about the vector properties, in Figure 5 the behaviors of the DOP of a focused twisted CGC radially polarized beam at different propagation distances for different values of $n$ is plotted. It has been shown that the on-axis DOP singularity of an SM radially polarized beam is propagation invariant [51]. However, for a twisted SM radially polarized beam, it is interesting to see from Figure 5a1-a3 that a similar on-axis DOP singularity disappears due to the existence of a twisted phase. In addition, quite different from the spectral density and the spectral DOC, a striking feature can be seen in which the twist phase does not lead to a rotation of the DOP.

Figure 6 illustrates the DOP and the corresponding cross line of a focused twisted CGC radially polarized beam at different propagation distances for different values of the twist phase. It is seen that the complex DOP structure gradually degenerates and becomes more uniform. This is because the astigmatism gradually increases with the increase of twist factor.
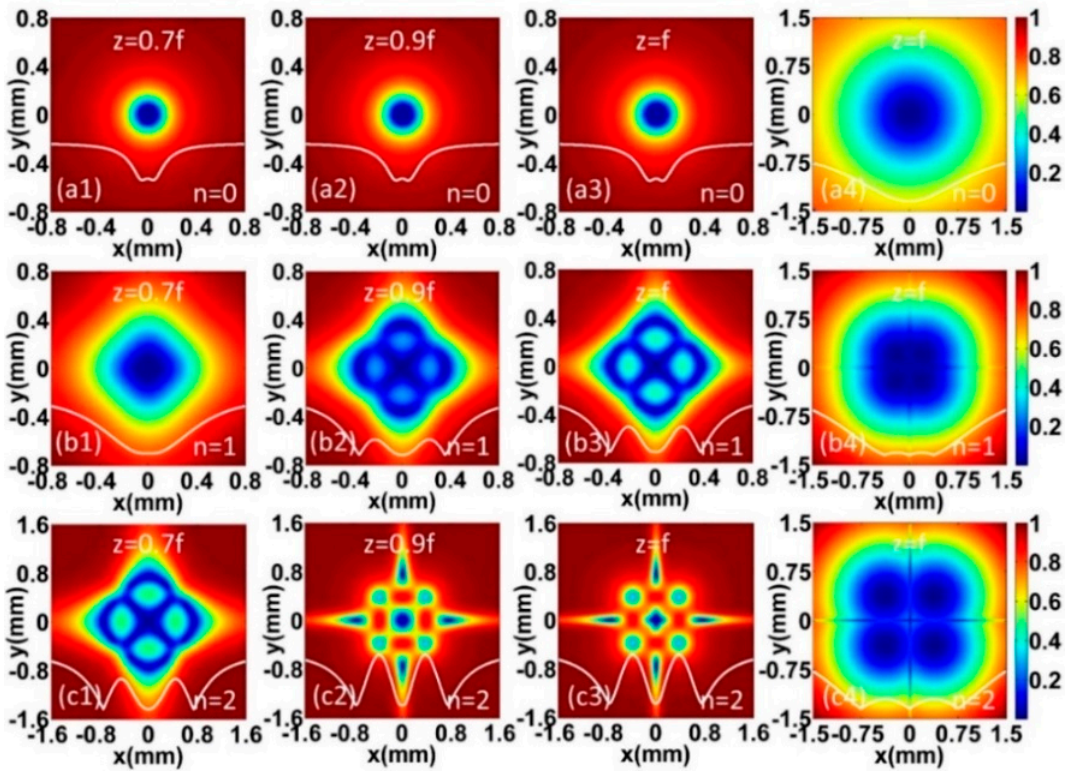

Figure 5. Density plot of the DOP and the corresponding cross line of a focused twisted CGC radially polarized beam at different propagation distances for different values of $n$. For (a1-a3), (b1-b3), (c1-c3) $\mu=0.001 \mathrm{~mm}^{-1} ;(\mathbf{a} 4, \mathbf{b} 4, \mathbf{c} 4) \mu=0 \mathrm{~mm}^{-1}$. 

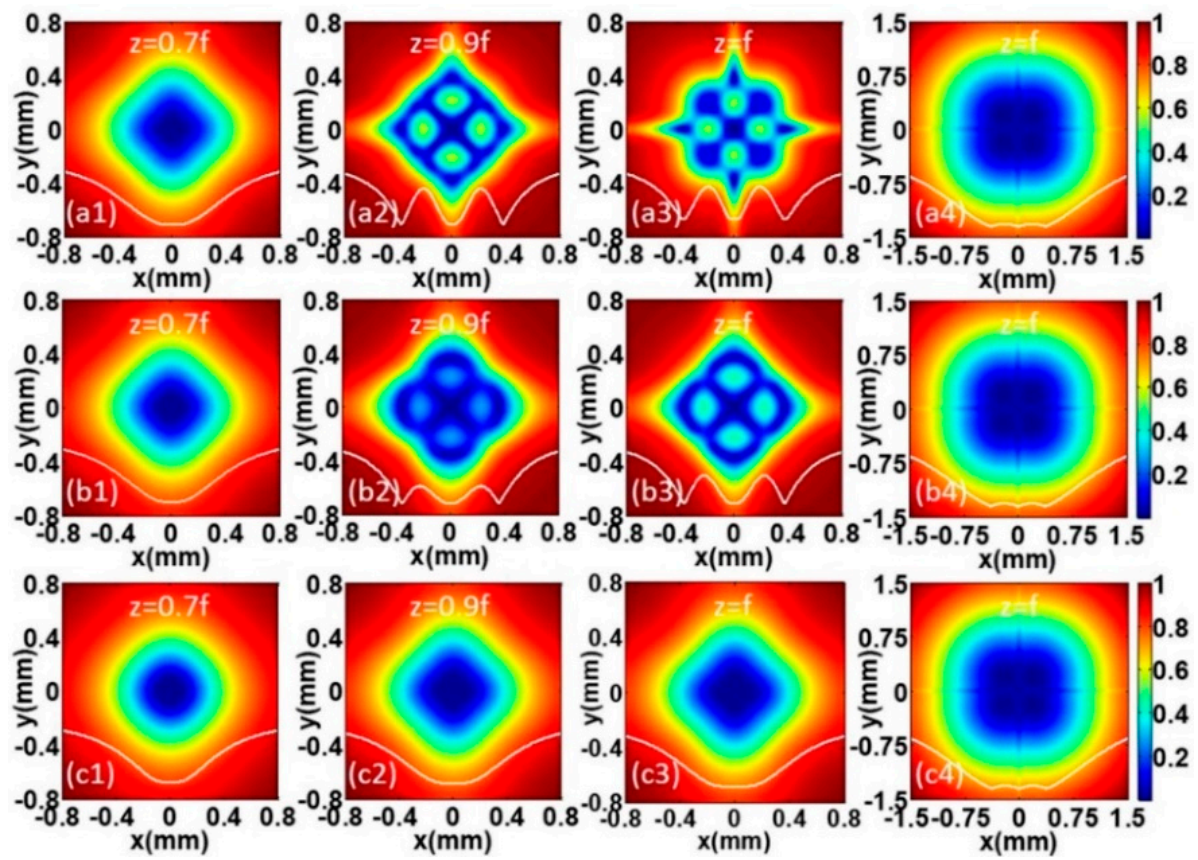

Figure 6. Density plot of the DOP the corresponding cross line of a focused twisted CGC radially polarized beam at different propagation distances for different values of the twist phase with $n=1$. For $(\mathbf{a 1}-\mathbf{a} 3) \mu=0.0005 \mathrm{~mm}^{-1}$; (b1-b3) $\mu=0.001 \mathrm{~mm}^{-1}$; (c1-c3) $\mu=0.002 \mathrm{~mm}^{-1}$; (a4,b4,c4) $\mu=0 \mathrm{~mm}^{-1}$.

Next, let us further evaluate the effects of the twist phase on the state of polarization (SOP). It is well known that the CSD matrix can be represented as a sum of a completely polarized beam and a completely unpolarized beam [2]. For a partially coherent beam, the polarization ellipse is a parameter characterizing the fully polarized portion of the beam. By using the CSD matrix, one can conveniently determine the polarization ellipse, including the major and minor semiaxes of the polarization ellipse, and the orientation angle [8]. The orientation angle $\varphi$ is given by the following formula:

$$
\varphi\left(\mathbf{r}^{\prime}\right)=\frac{1}{2} \arctan \left(\frac{2 \operatorname{Re}\left[W_{x y}\left(\mathbf{r}^{\prime}, \mathbf{r}^{\prime}\right)\right]}{W_{x x}\left(\mathbf{r}^{\prime}, \mathbf{r}^{\prime}\right)-W_{y y}\left(\mathbf{r}^{\prime}, \mathbf{r}^{\prime}\right)}\right), \quad(-\pi / 2 \leq \varphi \leq \pi / 2),
$$

and the major and minor semiaxes of the polarization ellipse take the following form:

$$
A_{ \pm}\left(\mathbf{r}^{\prime}, \mathbf{r}^{\prime}\right)=\frac{1}{\sqrt{2}}\left\{\sqrt{\left(W_{x x}-W_{y y}\right)^{2}+4\left|W_{x y}\right|^{2}} \pm \sqrt{\left(W_{x x}-W_{y y}\right)^{2}+4\left(\operatorname{Re}\left[W_{x y}\right]\right)^{2}}\right\}^{1 / 2} .
$$

Then, the degree of ellipticity $\varepsilon$ characterizing the shape of the polarization ellipse of an electromagnetic beam is defined by:

$$
\varepsilon=A_{-}\left(\mathbf{r}^{\prime}, \mathbf{r}^{\prime}\right) / A_{+}\left(\mathbf{r}^{\prime}, \mathbf{r}^{\prime}\right), \quad 0 \leq \varepsilon \leq 1 .
$$

On submitting from Equations (5)-(8) into Equations (13)-(15), we numerically investigated the behaviors of the SOP in Figure 7 of a focused twisted CGC radially polarized beam at different propagation distances for different values of $n$. Owing to the twisted phase, it is of interest to find from Figure 7a1-a4 that the initial radially polarized structure gradually evolves into an azimuthally polarized structure in the focal plane. Moreover, similar to the spectral DOC, one finds that the SOP experiences a rotation of $\pi / 2$ with the focal length, regardless of the value of order $n$. In addition, 
for the case of $n \neq 0$, the SOP splits with the splitting of the spectral intensity, which is the same as that in absence of the twist phase $[25,26]$.

Figure 8 shows the dependence of the SOP on the twist phase. Similar to the spectral density and the spectral DOC, it is seen that although the twist speed of the SOP closely depends on the value of the twist phase, the SOP always rotates $\pi / 2$ within the focal length compared to that without twist factor. Therefore, it is worthwhile to manipulate novel complex vector beams by taking advantage of the twist phase and tailored coherence structure.
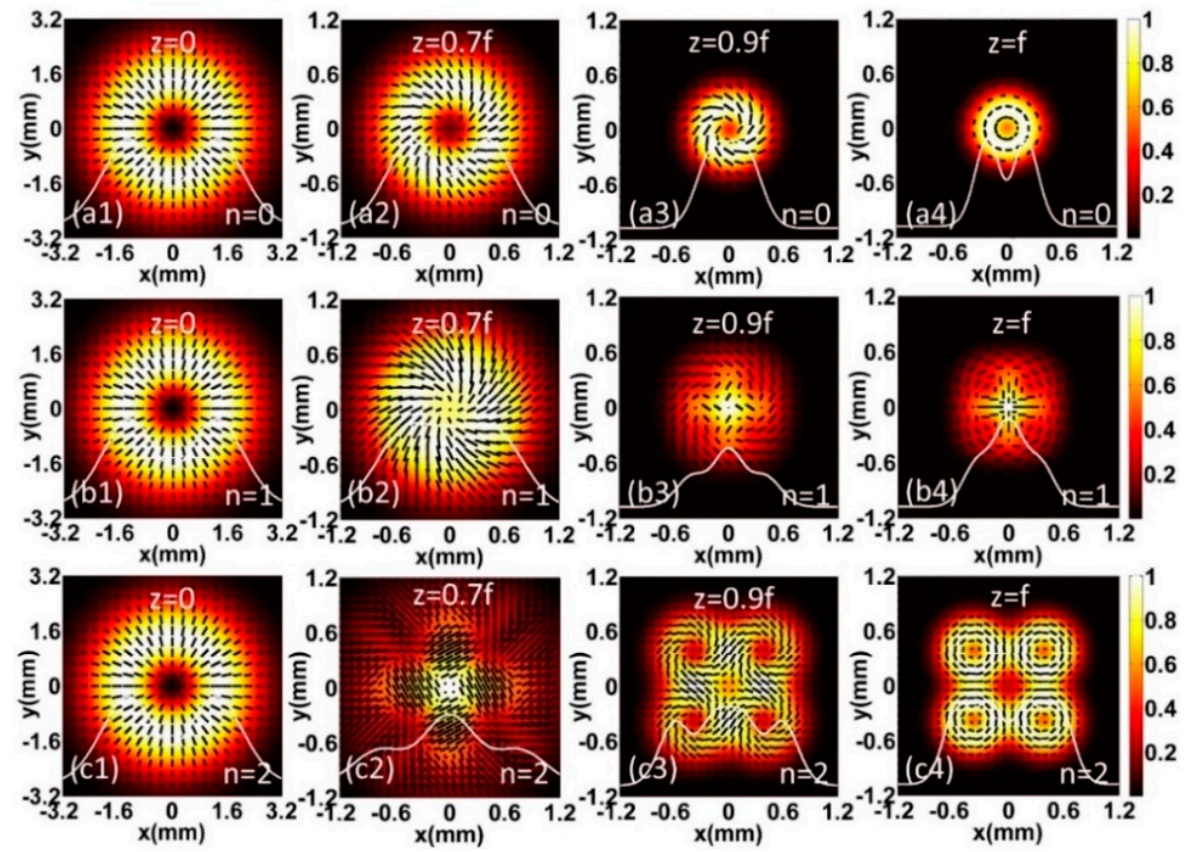

Figure 7. The state of polarization (SOP) and the corresponding cross line of a focused twisted CGC radially polarized beam at different propagation distances for different values of $n$. For (a1-a3), (b1-b3), (c1-c3) $\mu=0.001 \mathrm{~mm}^{-1} ;(\mathbf{a} 4, \mathrm{~b} 4, \mathrm{c} 4) \mu=0 \mathrm{~mm}^{-1}$.
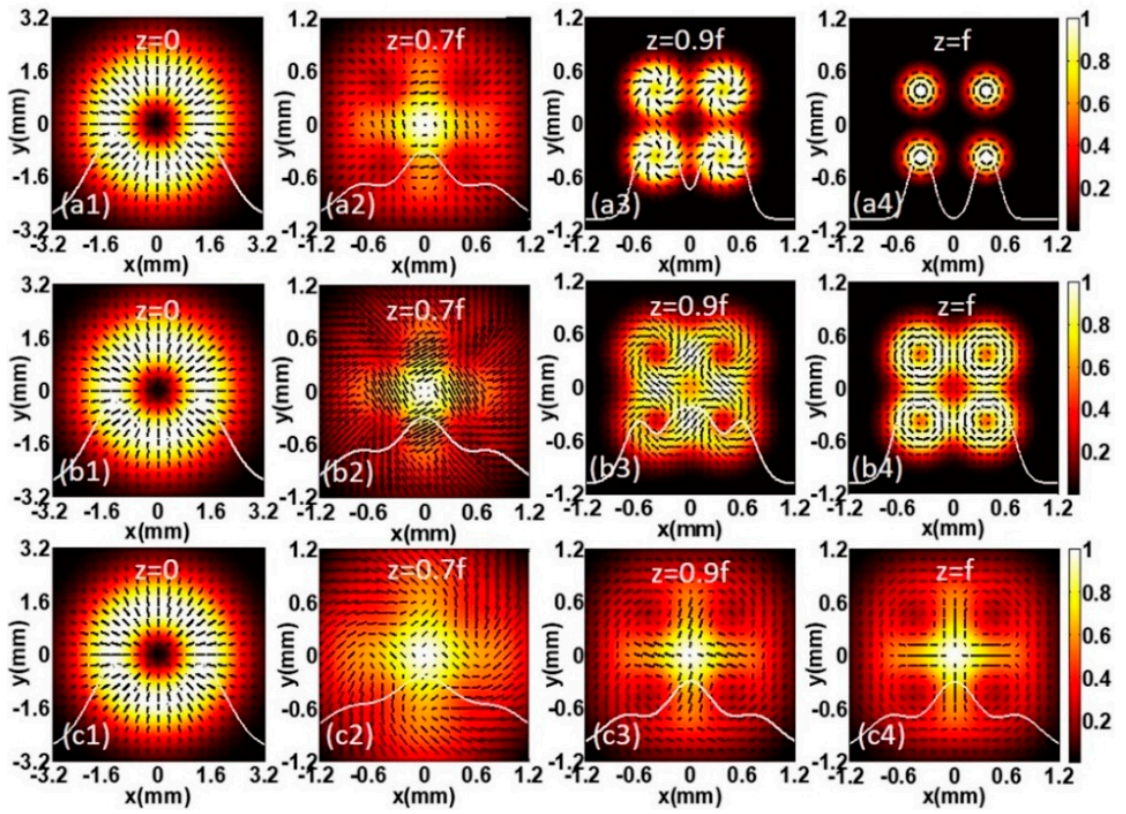

Figure 8. The SOP and the corresponding cross line of focused twisted CGC radially polarized beam at different propagation distances for different values of the twist phase with $n=2$. For (a1-a4) $\mu=0.0005 \mathrm{~mm}^{-1}$; (b1-b4) $\mu=0.001 \mathrm{~mm}^{-1}$; (c1-c4) $\mu=0.002 \mathrm{~mm}^{-1}$. 
Finally, we concentrate our attention on the spectral properties of a focused polychromatic twisted CGC radially polarized beam. It is well known that the correlation-induced spectral change (also called the "Wolf effect") is an important feature of partially coherent beams. It was demonstrated that Wolf effect has important applications in optical signal processing, information encoding and exchange $[5,6,67-70]$. Since the spectral change is closely related to the coherence and polarization, it is meaningful to study the spectral shift of a twisted nontrivial CGC radially polarized beam. Here, let us assume that the initial spectrum is a Lorentz type with $\omega_{0}$ being the central frequency and $\Gamma_{0}$ being the half-width at half-maximum, and $\omega$ is the angular frequency. Then the elements of the CSD matrix of a focused polychromatic twisted CGC radially polarized beam in the output plane are given as:

$$
\begin{aligned}
& W_{x x}\left(\mathbf{r}_{1}^{\prime}, \mathbf{r}_{2}^{\prime}, \omega\right)=\frac{k^{2}}{64 w_{0}^{2} B^{2} N_{1}^{2}\left(N_{2}-\Omega\right)^{2}} \cdot \frac{\Gamma_{0}^{2}}{\left[\left(\omega-\omega_{0}\right)^{2}+\Gamma_{0}^{2}\right]} \exp \left[-\frac{i k D}{2 B}\left(\mathbf{r}_{1}^{\prime 2}-\mathbf{r}_{2}^{\prime 2}\right)\right] \\
& \times \sum_{a_{1}= \pm a} \sum_{a_{2}= \pm a}\left[\xi_{u 11}\left(\xi_{u 21}+i \Pi_{1}\right)+\delta_{0}^{-2}-\frac{\left(i \xi_{u 21}-\Pi_{1}\right)^{2}}{2 \delta_{0}^{2}\left(N_{2}-\Omega\right)}+\frac{k \mu\left(\xi_{u 21}+i \Pi_{1}\right)\left(i \xi_{v 22}-\Pi_{2}\right)}{2\left(N_{2}-\Omega\right)}\right] \\
& \times \exp \left[-\frac{\xi_{v 12}^{2}+\tau_{u 11}^{2}}{4 N_{1}}+\frac{\left(i \xi_{u 21}-\Pi_{1}\right)^{2}+\left(i \xi_{v 22}-\Pi_{2}\right)^{2}}{4\left(N_{2}-\Omega\right)}\right] \text {, } \\
& W_{y y}\left(\mathbf{r}_{1}^{\prime}, \mathbf{r}_{2}^{\prime}, \omega\right)=\frac{k^{2}}{64 w_{0}^{2} B^{2} N_{1}^{2}\left(N_{2}-\Omega\right)^{2}} \cdot \frac{\Gamma_{0}^{2}}{\left[\left(\omega-\omega_{0}\right)^{2}+\Gamma_{0}^{2}\right]} \exp \left[-\frac{i k D}{2 B}\left(\mathbf{r}_{1}^{\prime 2}-\mathbf{r}_{2}^{\prime 2}\right)\right] \\
& \times \sum_{a_{1}= \pm a} \sum_{a_{2}= \pm a}\left[\xi_{v 12}\left(\xi_{v 22}+i \Pi_{2}\right)+\delta_{0}^{-2}-\frac{\left(i \xi_{v 22}-\Pi_{2}\right)^{2}}{2 \delta_{0}^{2}\left(N_{2}-\Omega\right)}-\frac{k \mu\left(\xi_{v 22}+i \Pi_{2}\right)\left(i \xi_{u 21}-\Pi_{1}\right)}{2\left(N_{2}-\Omega\right)}\right] \\
& \times \exp \left[-\frac{\tilde{\xi}_{v 12}^{2}+\tilde{\xi}_{u 11}^{2}}{4 N_{1}}+\frac{\left(i \tilde{\zeta}_{v 22}-\Pi_{2}\right)^{2}+\left(i \tilde{\xi}_{u 21}-\Pi_{1}\right)^{2}}{4\left(N_{2}-\Omega\right)}\right], \\
& W_{x y}\left(\mathbf{r}_{1}^{\prime}, \mathbf{r}_{2}^{\prime}, \omega\right)=\frac{k^{2}}{64 w_{0}^{2} B^{2} N_{1}^{2}\left(N_{2}-\Omega\right)^{2}} \cdot \frac{\Gamma_{0}^{2}}{\left[\left(\omega-\omega_{0}\right)^{2}+\Gamma_{0}^{2}\right]} \exp \left[-\frac{i k D}{2 B}\left(\mathbf{r}_{1}^{\prime 2}-\mathbf{r}_{2}^{\prime 2}\right)\right] \\
& \times \sum_{a_{1}= \pm a} \sum_{a_{2}= \pm a}\left[\xi_{u 11}\left(\xi_{v 22}+i \Pi_{2}\right)+i k \mu+i k \mu \frac{\left(i \xi_{v 22}-\Pi_{2}\right)^{2}}{2\left(N_{2}-\Omega\right)}-\frac{\left(i \xi_{v 22}-\Pi_{2}\right)\left(i \xi_{u 21}-\Pi_{1}\right)}{2 \delta_{0}^{2}\left(N_{2}-\Omega\right)}\right] \\
& \times \exp \left[-\frac{\xi_{v 12}^{2}+\tilde{\zeta}^{2} u 11}{4 N_{1}}+\frac{\left(i \xi_{v 22}-\Pi_{2}\right)^{2}+\left(i \tau_{u 21}-\Pi_{1}\right)^{2}}{4\left(N_{2}-\Omega\right)}\right] .
\end{aligned}
$$

Now, we numerically investigate the relative spectral changes of a polychromatic twisted CGC radially polarized beam focused by a thin lens. The spectral shift $\Delta \omega$ is the difference between the peak frequency $\omega_{\max }$ of the spectrum after propagation and the central frequency $\omega_{0}$ of the source spectrum. A positive value of $\Delta \omega$ means a blueshift, while a negative value denotes a redshift. The relative spectral shift can be defined as:

$$
\gamma=\left(\omega_{\max }-\omega_{0}\right) / \omega_{0}
$$

The parameters are set as $\omega_{0}=3.6 \times 10^{15}$ and $\Gamma_{0}=1 \times 10^{14}$. Figure 9 shows the relative spectral shift $\gamma$ of a focused polychromatic twisted CGC radially polarized beam versus the propagation distance $z$. For a polychromatic partially coherent radially polarized beam without the twist phase, a study showed that an on-axis blueshift can be found before and after the focus [47]. However, for a polychromatic twisted radially polarized beam, the on-axis spectral shift is always redshifted, with the minimum redshift occurring in the focal plane, see Figure 9e. With the increase of the beam order $n$, the blueshift appears and the maximum blueshift occurs in the focal plane. In addition, one also finds that the maximum redshift occurs off-axis in the focal plane and grows rapidly as the off-axis distance $y$ increases. In order to learn more about the effect of the twist phase on spectral shift, Figure $9 b-d$ plots the relative spectral shift of a focused polychromatic twisted CGC radially polarized beam versus the propagation distance for different values of the twist phase. One finds that the maximum value of redshift and blueshift are significantly reduced as the twist phase increases. The direct reason is that the divergence of the beam increases with the growth of the twist phase. As a result, the spectral density distribution becomes more uniform, leading to a gradual decrease in the difference in spectral shift. This is similar to the correlation-induced spectral change [2,5]. Moreover, different from a polychromatic radially polarized SM beam [47], one finds from Figure $9 f$ that there is 
a bimodal blueshift observed near the focal plane for a polychromatic CGC radially polarized beam with $n=1$. It is important to note that the effect of the twist phase on the spectral change is similar to the coherence, which is achieved by modulating the spectral density distribution upon propagation.
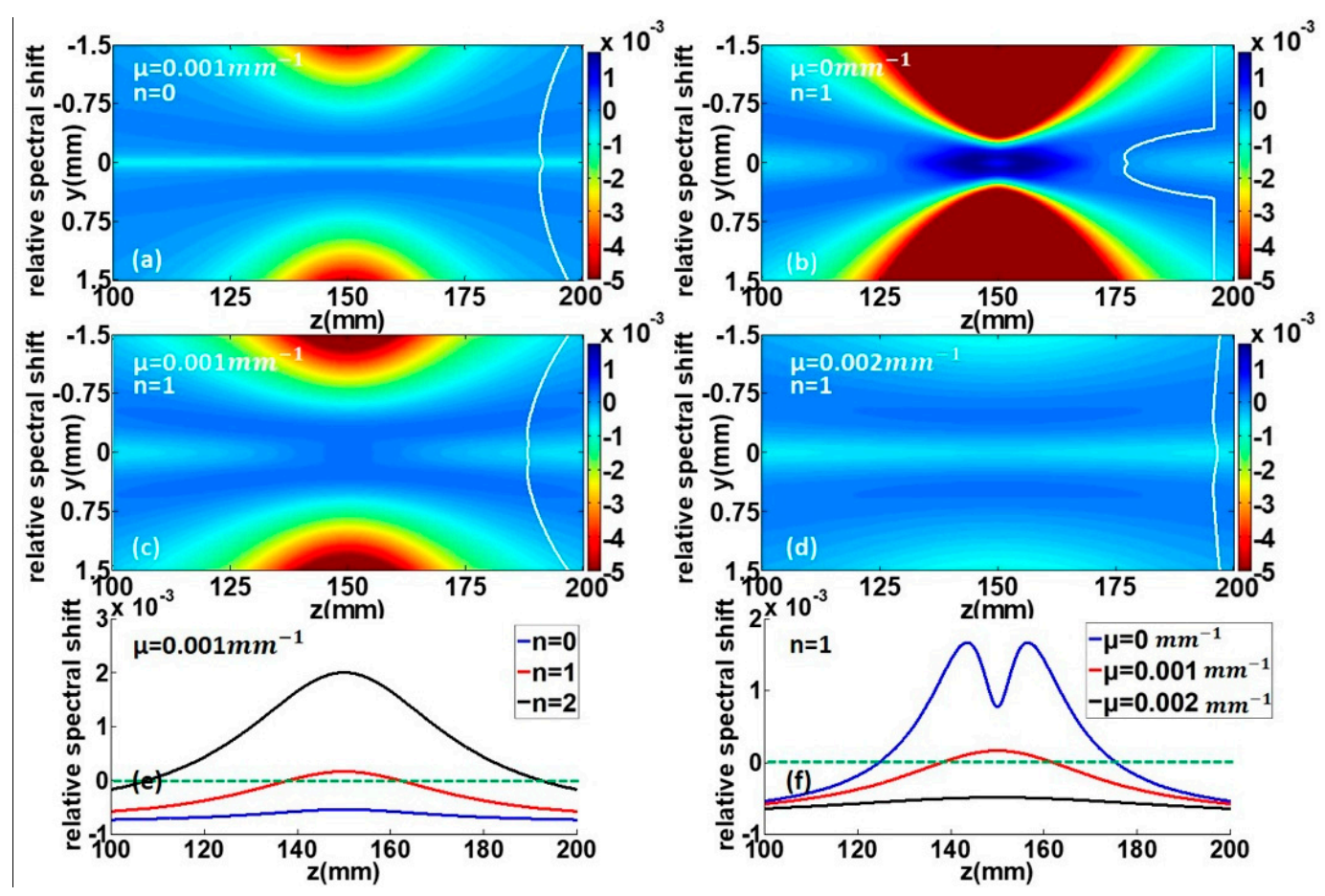

Figure 9. Relative spectral shift $\gamma$ of a focused polychromatic twisted CGC radially polarized beam versus the propagation distance and the ordinate $y$ with $x=0$, and relative on-axis spectral shift versus the propagation distance. For $(\mathbf{a}-\mathbf{d})$ contour plots of the relative spectral shift with $x=0$; $(\mathbf{e}, \mathbf{f})$ on-axis relative spectral shift with $x=0, y=0$.

\section{Conclusions}

As a summary, we have studied the focusing properties of a new class of twisted cosine-Gaussian correlated radially polarized beam. It is shown that the twist phase leads to an astigmatism of the light beam, thereby affecting the various statistical properties of the beam during spreading. Because of the twisted phase, the beam spot, the degree of coherence, and the state of polarization experience rotation during transmission, but the degree of polarization is not twisted. Meanwhile, although the rotation speed is nonlinear and closely depends on the value of the twist factor, they all undergo a rotation of $\pi / 2$ when they reach the focal plane. In addition, it turns out that the effect of the twist phase on the spectral change is essentially similar to coherence, which is achieved by modulating the spectral density distribution during transmission. These results provide a useful guideline for the adjustable twist phase to generate novel partially coherent vector beams and promote important potential applications in the field of beam shaping, optical tweezers, optical imaging, and free space optical communications.

Author Contributions: Data curation, J.Z.; Formal analysis, S.Z.; Funding acquisition, S.Z.; Investigation, S.Z. and H.H.; Resources, Z.L. and J.L.; Supervision, S.Z., J.W., H.W., Z.L. and J.L.; Validation, J.Z.; Writing一original draft, S.Z.; Writing—review \& editing, S.Z.

Funding: This research was funded by National Natural Science Foundation of China [11504172]; The China Postdoctoral Science Foundation [7131701018]; The Postdoctoral Science Foundation of Jiangsu Province [7131707317]; The Natural Science Foundation of Jiangsu Province [BK20150763]; The Fundamental Research Funds for the Central Universities (30917011336, 30916014112-001), and the Jiangsu Overseas Visiting Scholar Program for University Prominent Young \& Middle-aged Teachers and Presidents. 
Conflicts of Interest: The authors declare no conflict of interest.

\section{References}

1. Mandel, L.; Wolf, E. Optical Coherence and Quantum Optics; Cambridge University: Cambridge, UK, 1995.

2. Wolf, E. Introduction to the Theory of Coherence and Polarization of Light; Cambridge University: Cambridge, UK, 2007.

3. Martínez-Herrero, R.; Mejías, P.M.; Piquero, G. Characterization of Partially Polarized Light Fields; Springer Science \& Business Media: Berlin, Germany, 2009.

4. Friberg, A.T.; Sudol, R.J. Propagation parameters of Gaussian Schell-model beams. Opt. Commun. 1982, 41, 383-387. [CrossRef]

5. Wolf, E. Invariance of the Spectrum of Light on Propagation. Phys. Rev. Lett. 1986, 56, 1370-1372. [CrossRef] [PubMed]

6. Pu, J.; Zhang, H.; Nemoto, S. Spectral shifts and spectral switches of partially coherent light passing throug an aperture. Opt. Commun. 1999, 162, 57-63. [CrossRef]

7. James, D.F.V. Changes of polarization of light beams on propagation in free space. J. Opt. Soc. Am. A 1994, 11, 1641-1643. [CrossRef]

8. Korotkova, O.; Wolf, E. Changes in the state of polarization of a random electromagnetic beam on propagation. Opt. Commun. 2005, 246, 35-43. [CrossRef]

9. Tervo, J.; Setälä, T.; Friberg, A.T. Degree of coherence for electromagnetic fields. Opt. Express 2003, 11, 1137-1143. [CrossRef] [PubMed]

10. Ellis, J.; Dogariu, A.; Ponomarenko, S.A.; Wolf, E. Correlation matrix of a completely polarized, statistically stationary electromagnetic field. Opt. Lett. 2004, 29, 1536-1538. [CrossRef] [PubMed]

11. Gori, F.; Santarsiero, M. Devising genuine spatial correlation functions. Opt. Lett. 2007, 32, 3531-3533. [CrossRef] [PubMed]

12. Gori, F.; Sanchez, V.R.; Santarsiero, M.; Shirai, T. On genuine cross-spectral density matrices. J. Opt. A 2009, 11, 085706. [CrossRef]

13. Borghi, R. Superposition scheme for $\mathrm{J}_{0}$-Correlated partially coherent sources. IEEE J. Quantum Electron. 2002, 35, 65-130.

14. Ponomarenko, S.A. A class of partially coherent beams carrying optical vortices. J. Opt. Soc. Am. A 2001, 18, 150-156. [CrossRef]

15. Lajunen, H.; Saastamoinen, T. Propagation characteristics of partially coherent beams with spatially varying correlations. Opt. Lett. 2011, 36, 4104-4106. [CrossRef] [PubMed]

16. Sahin, S.; Korotkova, O. Light sources generating far fields with tunable flat profiles. Opt. Lett. 2012, 37, 2970-2972. [CrossRef] [PubMed]

17. Tong, Z.; Korotkova, O. Non-uniformly correlated light beams in uniformly correlated media. Opt. Lett. 2012, 37, 3240-3242. [CrossRef] [PubMed]

18. Mei, Z.; Korotkova, O. Random sources generating ring-shaped beams. Opt. Lett. 2013, 38, 91-93. [CrossRef] [PubMed]

19. Cai, Y.; Chen, Y.; Wang, F. Generation and propagation of partially coherent beams with non-conventional correlation functions: A review. J. Opt. Soc. Am. A 2014, 31, 2083-2096. [CrossRef] [PubMed]

20. Wang, F.; Liu, X.; Yuan, Y.; Cai, Y. Experimental generation of partially coherent beams with different complex degrees of coherence. Opt. Lett. 2013, 38, 1814-1816. [CrossRef] [PubMed]

21. Chen, Y.; Gu, J.; Wang, F.; Cai, Y. Self-splitting properties of a Hermite-Gaussian correlated Schell-model beam. Phys. Rev. A 2014, 91, 013832. [CrossRef]

22. Ma, L.; Ponomarenko, S.A. Optical coherence gratings and lattices. Opt. Lett. 2014, 39, 6656-6659. [CrossRef] [PubMed]

23. Ma, L.; Ponomarenko, S.A. Free-space propagation of optical coherence lattices and periodicity reciprocity. Opt. Express 2015, 23, 1848-1856. [CrossRef] [PubMed]

24. Mei, Z.; Zhao, D.; Korotkova, O. Gaussian Schell-model arrays. Opt. Lett. 2015, 40, 5662-5665. [CrossRef] [PubMed]

25. Zhu, S.; Chen, Y.; Wang, J.; Wang, H.; Li, Z.; Cai, Y. Generation and propagation of a vector cosine-Gaussian correlated beam with radial polarization. Opt. Express 2015, 23, 33099-33115. [CrossRef] [PubMed] 
26. Zhu, S.; Wang, J.; Liu, X.; Cai, Y.; Li, Z. Generation of arbitrary radially polarized array beams by manipulating correlation structure. Appl. Phys. Lett. 2016, 109, 161904. [CrossRef]

27. Liu, X.; Yu, J.; Cai, Y.; Ponomarenko, S.A. Propagation of optical coherence lattices in the turbulent atmosphere. Opt. Lett. 2016, 41, 4182-4185. [CrossRef] [PubMed]

28. Wang, J.; Zhu, S.; Li, Z. Vector properties of a tunable random electromagnetic beam in non-Kolmogrov turbulence. Chin. Opt. Lett. 2016, 14, 080101. [CrossRef]

29. Santarsiero, M.; Martínez-Herrero, R.; Maluenda, D.; de Sande, J.C.G.; Piquero, G.; Gori, F. Partially coherent sources with circular coherence. Opt. Lett. 2017, 42, 1512-1515. [CrossRef] [PubMed]

30. Ding, C.; Koivurova, M.; Turunen, J.; Pan, L. Self-focusing of a partially coherent beam with circular coherence. J. Opt. Soc. Am. A 2017, 34, 1441-1447. [CrossRef] [PubMed]

31. Wang, Z.; Wang, J.; Huang, L.; Zhu, S.; Li, Z. Propagation of a vector cosine-Gaussian correlated beam through an active GRIN medium. IEEE Photon. J. 2017, 7908312. [CrossRef]

32. Wan, L.; Zhao, D. Optical coherence grids and their propagation characteristics. Opt. Express 2018, 26, 2168-2180. [CrossRef] [PubMed]

33. Liang, C.; Zhu, X.; Mi, C.; Peng, X.; Wang, F.; Cai, Y.; Ponomarenko, S.A. High-quality partially coherent Bessel beam array generation. Opt. Lett. 2018, 43, 3188-3191. [CrossRef] [PubMed]

34. Youngworth, K. S.; Brown, T.G. Focusing of high numerical aperture cylindrical-vector beams. Opt. Express 2000, 7, 77-87. [CrossRef] [PubMed]

35. Dorn, R.; Quabis, S.; Leuchs, G. Sharper focus for a radially polarized light beam. Phys. Rev. Lett. 2003, 91, 233901. [CrossRef] [PubMed]

36. Kozawa, Y.; Sato, S. Generation of a radially polarized laser beam by use of a conical Brewster prism. Opt. Lett. 2005, 30, 3063-3065. [CrossRef] [PubMed]

37. Deng, D.; Guo, Q. Analytical vectorial structure of radially polarized light beams. Opt. Lett. 2007, 32, 2711-2713. [CrossRef] [PubMed]

38. Wang, H.; Shi, L.; Lukyanchuk, B.; Sheppard, C.J.R.; Chong, C.T. Creation of a needle of longitudinally polarized light in vacuum using binary optics. Nat. Photonics 2008, 2, 501-505. [CrossRef]

39. Wróbel, P.; Pniewski, J.; Antosiewicz, T.J.; Szoplik, T. Focusing radially polarized light by a concentrically corrugated silver film without a hole. Phys. Rev. Lett. 2009, 102, 183902. [CrossRef] [PubMed]

40. Chen, W.; Abeysinghe, D.C.; Nelson, R.L.; Zhan, Q. Plasmonic lens made of multiple concentric metallic rings under radially polarized illumination. Nano Lett. 2009, 9, 4320-4325. [CrossRef] [PubMed]

41. Gu, Y.; Korotkova, O.; Gbur, G. Scintillation of nonuniformly polarized beams in atmospheric turbulence. Opt. Lett. 2009, 34, 2261-2263. [CrossRef] [PubMed]

42. Li, X.; Lan, T.H.; Tien, C.H.; Gu, M. Three-dimensional orientation-unlimited polarization encryption by a single optically configured vectorial beam. Nat. Commun. 2012, 3, 998. [CrossRef] [PubMed]

43. Dong, Y.; Feng, F.; Chen, Y.; Zhao, C.; Cai, Y. Statistical properties of a nonparaxial cylindrical vector partially coherent field in free space. Opt. Express 2012, 20, 15908-15927. [CrossRef] [PubMed]

44. Wu, G.; Wang, F.; Cai, Y. Coherence and polarization properties of a radially polarized beam with variable spatial coherence. Opt. Express 2012, 20, 28301-28318. [CrossRef] [PubMed]

45. Chen, R.; Dong, Y.; Wang, F.; Cai, Y. Statistical properties of a cylindrical vector partially coherent beam in turbulent atmosphere. Appl. Phys. B 2013, 112, 247-259. [CrossRef]

46. Wang, F.; Cai, Y.; Dong, Y.; Korotkova, O. Experimental generation of a radially polarized beam with controllable spatial coherence. Appl. Phys. Lett. 2012, 100, 051108. [CrossRef]

47. Zhu, S.; Zhu, X.; Liu, L.; Wang, F.; Cai, Y. Theoretical and experimental studies of the spectral changes of a polychromatic partially coherent radially polarized beam. Opt. Express 2013, 21, 27682-27696. [CrossRef] [PubMed]

48. Zhu, S.; Wang, F.; Chen, Y.; Li, Z.; Cai, Y. Statistical properties in Young's interference pattern formed with a radially polarized beam with controllable spatial coherence. Opt. Express 2014, 22, 28697-28710. [CrossRef] [PubMed]

49. Martínez-Herrero, R.; Prado, F. Polarization evolution of radially polarized partially coherent vortex fields: role of Gouy phase of Laguerre-Gauss beams. Opt. Express 2015, 23, 5043-5051. [CrossRef] [PubMed]

50. Wang, F.; Liu, X.; Liu, L.; Yuan, Y.; Cai, Y. Experimental study of the scintillation index of a radially polarized beam with controllable spatial coherence. Appl. Phys. Lett. 2013, 103, 091102. [CrossRef] 
51. Wang, J.; Zhu, S.; Wang, H.; Cai, Y.; Li, Z. Second-order statistics of a radially polarized cosine-Gaussian correlated Schell-model beam in anisotropic turbulence. Opt. Express 2016, 24, 11626-11639. [CrossRef] [PubMed]

52. Simon, R.; Mukunda, N. Twisted Gaussian Schell-model beams. J. Opt. Soc. Am. A 1993, 10, 95-109. [CrossRef]

53. Friberg, A.T.; Tervonen, E.; Turunen, J. Interpretation and experimental demonstration of twisted Gaussian Schell-model beams. J. Opt. Soc. Am. A 1994, 11, 1818-1826. [CrossRef]

54. Borghi, R.; Gori, F.; Guattari, G.; Santarsiero, M. Twisted Schell-model beams with axial symmetry. Opt. Lett. 2015, 40, 4504-4507. [CrossRef] [PubMed]

55. Gori, F.; Santarsiero, M. Devising genuine twisted cross-spectral densities. Opt. Lett. 2018, 43, 595-598. [CrossRef] [PubMed]

56. Ambrosini, D.; Bagini, V.; Gori, F.; Santarsiero, M. Twisted Gaussian Schell-model beams: A superposition model. J. Mod. Opt. 1994, 41, 1391-1399. [CrossRef]

57. Simon, R.; Friberg, A.T.; Wolf, E. Transfer of radiance by twisted Gaussian Schell-model beams in paraxial systems. Pure Appl. Opt. 1996, 5, 331-343. [CrossRef]

58. Ponomarenko, S.A. Twisted Gaussian Schell-model solitons. Phys. Rev. E 2001, 64, 036618. [CrossRef] [PubMed]

59. Lin, Q.; Cai, Y. Tensor ABCD law for partially coherent twisted anisotropic Gaussian-Schell model beams. Opt. Lett. 2002, 27, 216-218. [CrossRef] [PubMed]

60. Zhu, S.; Cai, Y. Spectral shift of a twisted electromagnetic Gaussian Schell-model beam focused by a thin lens. App. Phys. B 2010, 99, 317-323. [CrossRef]

61. Zhao, C.; Cai, Y.; Korotkova, O. Radiation force of scalar and electromagnetic twisted Gaussian Schell-model beams. Opt. Express 2009, 17, 21472-21487. [CrossRef] [PubMed]

62. Wang, F.; Cai, Y. Second-order statistics of a twisted Gaussian Schell-model beam in turbulent atmosphere. Opt. Express 2010, 18, 24661-24672. [CrossRef] [PubMed]

63. Wang, F.; Cai, Y.; Eyyuboğlu, H.T.; Baykal, Y. Twist phase-induced reduction in scintillation of a partially coherent beam in turbulent atmosphere. Opt. Lett. 2015, 40, 4504-4507. [CrossRef] [PubMed]

64. Wang, J.; Wang, H.; Zhu, S.; Li, Z. Second-order moments of a twisted Gaussian Schell-model beam in anisotropic turbulence. J. Opt. Soc. Am. A 2018, 35, 1173-1179. [CrossRef] [PubMed]

65. Serna, J.; Movilla, J.M. Orbital angular momentum of partially coherent beams. Opt. Lett. 2001, 26, $405-407$. [CrossRef] [PubMed]

66. Cai, Y.; Zhu, S. Orbital angular moment of a partially coherent beam propagating through an astigmatic ABCD optical system with loss or gain. Opt. Lett. 2014, 39, 1968-1971. [CrossRef] [PubMed]

67. Kandpal, H.C.; Vaishya, J.S.; Joshi, K.C. Wolf shift and its application in spectroradiometry. Opt. Commun. 1989, 73, 169-172. [CrossRef]

68. James, D.F.; Kandpal, H.C.; Wolf, E. A new method for determining the angular separation of double stars. Astrophys. J. 1995, 445, 406-410. [CrossRef]

69. Zhu, S.; Zhao, C.; Chen, Y.; Cai, Y. Experimental generation of a polychromatic partially coherent dark hollow beam. Optik 2013, 124, 5271-5273. [CrossRef]

70. Zhu, S.; Li, Z. Theoretical and experimental studies of the spectral changes of a focused polychromatic partially coherent flat-topped beam. Appl. Phys. B 2015, 118, 481-487. [CrossRef]

(C) 2018 by the authors. Licensee MDPI, Basel, Switzerland. This article is an open access article distributed under the terms and conditions of the Creative Commons Attribution (CC BY) license (http://creativecommons.org/licenses/by/4.0/). 